\title{
Anisotropic spin-orbit interaction revealed by in-plane magnetoresistance in single-oriented $\mathrm{SrRuO}_{3}$ thin films
}

\author{
R. Gunnarsson* \\ MC2, Chalmers University of Technology, SE-41296 Göteborg, Sweden and HLK, Jönköping University, SE-551 11 Jönköping, Sweden
}

(Received 20 December 2011; revised manuscript received 20 March 2012; published 5 June 2012)

\begin{abstract}
A detailed analysis of the in-plane magnetoresistance anisotropy in single-orientation thin-film $\mathrm{SrRuO}_{3}$ grown on (001) $\mathrm{SrTiO}_{3}$ is presented. The resistivity is measured in strips along [001], [1110], and [111 $]$ ] with an in-plane rotating magnetic field. The data show additional details in the transfer from negative to positive magnetoresistance when the field is rotated in the plane. They show that the magnetoresistance is anisotropic with respect to the crystalline directions rather than with respect to the direction of the current. The data suggest an anisotropic spin-orbit interaction and can possibly be a sign of in-plane weak antilocalization.
\end{abstract}

DOI: 10.1103/PhysRevB.85.235409

PACS number(s): 71.27.+a, 72.15.Rn, 75.70.Tj

\section{INTRODUCTION}

The spin-orbit interaction has been, and still is, an intriguing effect. Its roots are deep down in relativistic quantum mechanics, and its applications give prospects to manipulate and control the spin of conduction electrons. ${ }^{1}$ In itinerant ferromagnetic systems magnetoresistance measurements offer a direct method for measurements of the spin-orbit interaction through weak localization. ${ }^{2}$ Weak localization can be described as interference among the conduction-electron wave functions in an electron-electron scattering event. In the presence of spin-orbit interaction the spin of the conduction electron can be rotated in a scattering event, which results in a destructive interference, an effect known as weak antilocalization. This shows up as a positive magnetoresistance at fields below a critical field related to the spin-orbit scattering time. ${ }^{3,4}$ At higher fields the negative contribution from weak localization to the magnetoresistance dominates.

A frequently studied ferromagnetic system with an itinerant exchange mechanism is the $4 d$ transition metal oxide $\mathrm{SrRuO}_{3}$. $\mathrm{SrRuO}_{3}$ has been considered to be a "bad metal" (with mean free path comparable to the interatomic spacing), ${ }^{5,6}$ but is a comparatively good conductor $(\sim 10 \mu \Omega \mathrm{cm})$ and is frequently used as electrode material in various applications of oxide electronic devices. ${ }^{7,8}$ However, more recent reports on quantum oscillations in $\mathrm{SrRuO}_{3}$ thin films, which can been taken as a sign of Fermi liquid behavior, ${ }^{9,10}$ suggest that the mean free path is considerably larger. In addition, this system has been shown to exhibit the anomalous Hall effect $^{1-13}$ as well as weak localization ${ }^{14}$-indications of quantum behavior of conduction electrons. Together with the reported high anisotropy field ${ }^{15}(\sim 10 \mathrm{~T})$ and ferromagnetic resonance measurements, ${ }^{16}$ both consistent with strong spinorbit coupling, one could then anticipate also the presence of signatures of weak antilocalization.

Actually, in the literature one can find evidence for a positive magnetoresistance in $\mathrm{SrRuO}_{3}$ thin films. In Refs. 6 and 17 a positive in-plane magnetoresistance is found when a magnetic field is applied perpendicular to the current (although it does not show any sign of a maximum), whereas with the field parallel to the current the magnetoresistance is negative. However, in two more recent papers ${ }^{18,19}$ the in-plane magnetoresistance shows a tendency to saturation.
The aim of the present study is to make a detailed analysis of the anisotropic in-plane magnetoresistance in thin-film $\mathrm{SrRuO}_{3}$ (SRO). It is shown that these systems can exhibit features characteristic for spin-orbit scattering, but that the positive magnetoresistance with its maximum appears to be anisotropic and observed only when the field is along the $[001]_{\text {SRO }}$ direction.

\section{EXPERIMENTAL DETAILS}

As-delivered $\mathrm{SrTiO}_{3}$ substrates were prepared with HF etching and annealing according to the procedure described, e.g., in Ref. 20 to achieve $\mathrm{TiO}_{2}$-terminated atomically flat surfaces. However, the nominally (001)-directed $\mathrm{SrTiO}_{3}$ (STO) substrates were unintentionally miscut by the vendor, giving an angle of on average $0.15^{\circ}$ between the substrate surface and $(001)_{\text {STO }}$. The substrates were scanned in an atomic force microscope and only substrates with unit-cell-high surface steps with edges parallel to $(100)_{\text {STO }}$ or the equivalent $(010)_{\text {STO }}$ were selected for this study; see Fig. 1(a). $\mathrm{SrRuO}_{3}$ thin films were deposited on the substrates. For the deposition conventional pulsed laser ablation with an excimer laser ( $\lambda=248 \mathrm{~nm}, \tau \approx 20 \mathrm{~ns}$ ) was used.

The structure of the films was examined on a large scale by x-ray diffraction (see Fig. 2), and on a local scale by transmission electron microscopy. ${ }^{21}$ The films generally showed a high crystalline quality, but with some differences particularly in the amount of twinning. In this paper only data from the film with highest quality (single orientation) are reported. However, the same kind of anisotropy has been observed in several other samples. In all samples the direction of the $\mathrm{SrRuO}_{3}$ unit cell in relation to the $\mathrm{SrTiO}_{3}$ substrate surface steps was found to be as shown in Fig. 1(b), which agrees well with previous studies. ${ }^{22-24}$

From the diffraction data, shown in Fig. 2, one can draw the conclusion that the $\mathrm{SrRuO}_{3}$ film selected for this study is very close to a completely single orientation. There is a peak from the $440_{\text {SRO }}$ reflection and, hidden under the substrate peak, one from the $\overline{4} \overline{4} 0_{\text {SRO }}$ reflection. In the $2 \theta / \omega$ - $\omega$ map there is a strong peak from the $112_{\text {SRO }}$ reflection, and next to the major film peak we observe a small trace from the $020_{\text {SRO }}$ reflection. The full width at half maximum (FWHM) of the $112_{\text {SRO }}$ film reflection is about $0.11^{\circ}$, compared to a FWHM 

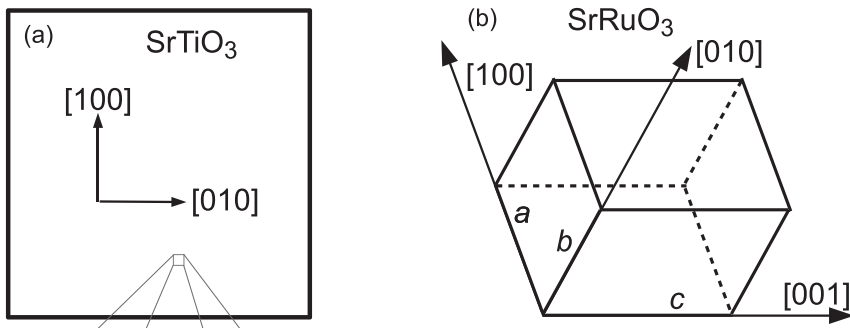

$1 \mu \mathrm{m} \times 1 \mu \mathrm{m}$

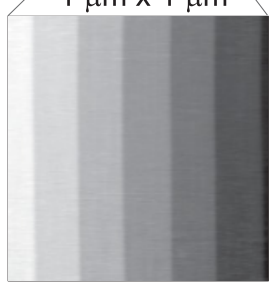

FIG. 1. (a) Schematic of a $\mathrm{SrTiO}_{3}$ substrate with atomic force microscope height image of a $1 \times 1 \mu \mathrm{m}^{2}$ area of a $\mathrm{TiO}_{2}$ substrate. The height difference from right (black) to left (light gray) is approximately $2.8 \mathrm{~nm}$, which corresponds to seven unit-cell-high steps on the $\mathrm{SrTiO}_{3}(a \sim 3.9 \AA)$ surface. (b) Schematic of the $\mathrm{SrRuO}_{3}$ unit cell and (below) its orientation in relation to the atomic steps on the $\mathrm{SrTiO}_{3}$ surface. The substrate steps are taken to have edges along the $[100]_{\text {STO }}$ direction.

of $0.006^{\circ}$ for the the $011_{\text {STO }}$ substrate reflection. By taking into account the peak volumes and the structure factors for the 112 and 020 reflections, respectively, one can estimate that at
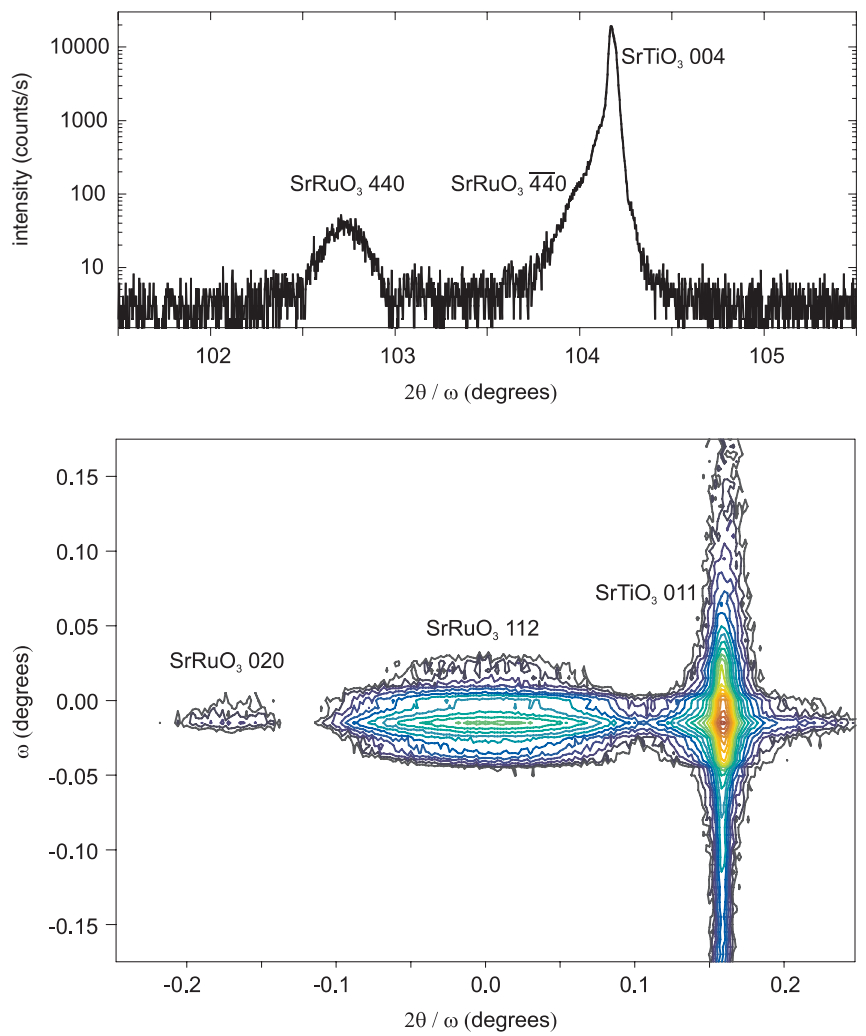

FIG. 2. (Color online) Top: X-ray $2 \theta / \omega$ diffraction data from the vicinity of the $\mathrm{SrTiO}_{3} 004$ reflection. Two film reflections can be seen: 440 and $\overline{4} \overline{4} 0$. Bottom: X-ray area scan $(2 \theta / \omega-\omega)$ around the $\mathrm{SrTiO}_{3}$ substrate 011 reflection and the $\mathrm{SrRuO}_{3} 112$ reflection.
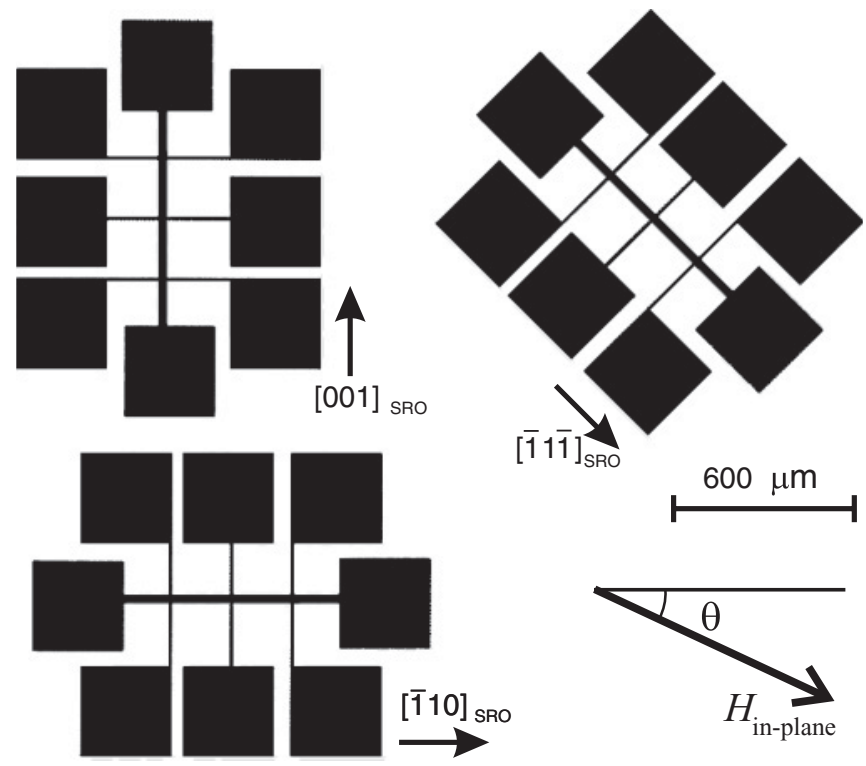

FIG. 3. Sketch of the alignment of Hall bars with respect to the $\mathrm{SrRuO}_{3}$ crystalline directions and the reference direction $\theta$ for the in-plane magnetic field $H$. The in-plane field direction is illustrated with the arrow to the lower right; $\theta=0^{\circ}$ and $\theta=90^{\circ}$ correspond to $[\overline{1} 10]_{\mathrm{SRO}}$ and $[00 \overline{1}]_{\mathrm{SRO}}$, respectively.

least $99.4 \%$ of the film has the orientation shown in Fig. 1(b). However, these detailed diffraction data were obtained from the patterned sample which could also have an impact on the degree of twinning observed.

The films were patterned by photolithography and Arion etching. Atomic steps were observed by atomic force microscopy on the sample surfaces even after the patterning processes. Somewhat different patterns were used, and in this paper results from a sample patterned with "Hall bars," with widths $30 \mu \mathrm{m}$ and lengths $200 \mu \mathrm{m}$, as shown in Fig. 3, are presented. The Hall bars were aligned in different directions with respect to the $\mathrm{SrRuO}_{3}$ unit-cell directionalong $[001]_{\mathrm{SRO}},[\overline{1} 10]_{\mathrm{SRO}}$, and $[\overline{1} 1 \overline{1}]_{\mathrm{SRO}}$, respectively. The film thickness is $50 \mathrm{~nm}$. Hence, by these preparations we achieved highly oriented $\mathrm{SrRuO}_{3}$ where it is possible to probe the electric properties along well-defined crystalline directions.

The electric transport properties were measured in a variable-temperature He-flow cryostat with a superconducting solenoid allowing a maximum field of $5 \mathrm{~T}$. For longitudinal resistance the sample was rotated in such a way that the magnetic field direction was varied in plane as shown in the lower right part of Fig. 3. [1110] is taken as the reference direction. For Hall effect measurements the field was applied perpendicular to the plane of the film.

\section{RESULTS}

\section{A. Temperature dependence of resistance}

The temperature dependence of the resistivity measured during slow heating after zero-field cooling is shown in Fig. 4. There is a kink in the resistance at $145 \mathrm{~K}$, typically taken as a token of the Curie temperature $T_{C}$. There is a small difference in the resistance for the two perpendicular crystallographic directions [110] and [001]. The resistance is slightly higher in 


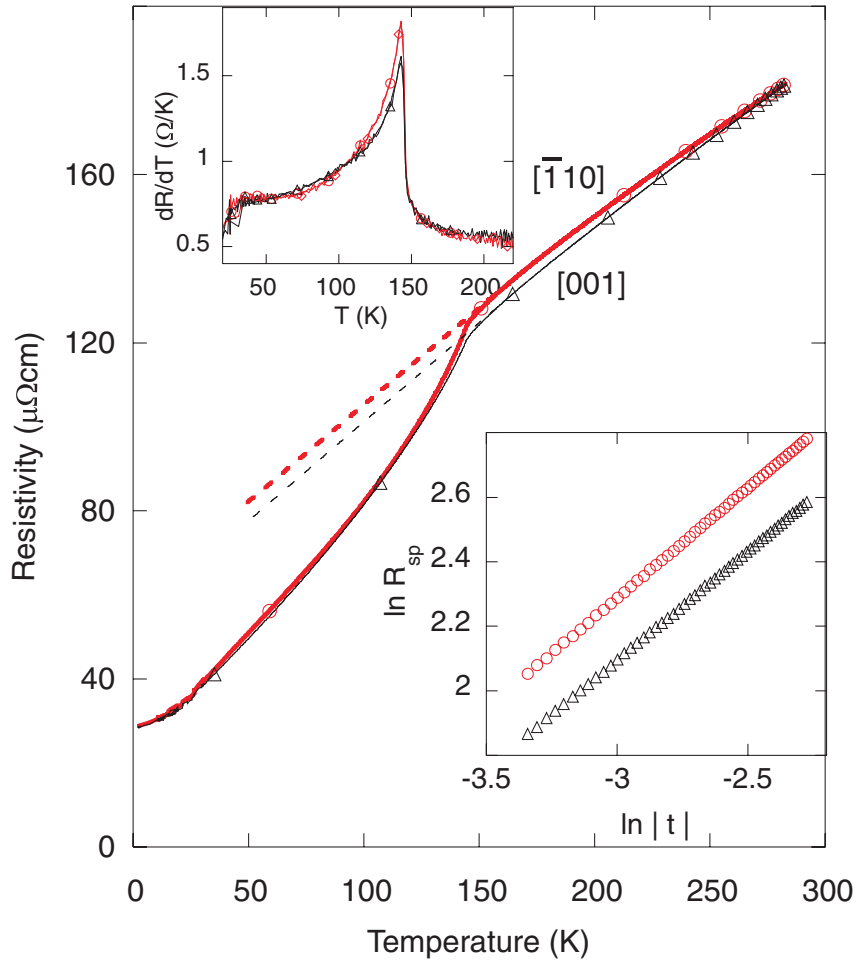

FIG. 4. (Color online) Temperature dependence of resistivity of the strips along the [110] (red line with circles) and the [001] (black line with triangles) directions. The dashed lines represent linear fits to the resistance data just above the Curie temperature. The upper inset shows the derivative of the resistance. The critical behavior of the spin-polarized resistance $\Delta \rho_{\mathrm{sp}}$ (details in the text) is shown in the inset to the lower right.

the [110] direction, in particular just above $T_{C}$, which agrees well with previously reported data. ${ }^{25}$ The low-temperature resistivity $\rho$ is approaching $28 \mu \Omega \mathrm{cm}$, or correspondingly the conductivity is about $\sigma=1 / \rho \approx 3.6 \times 10^{4}(\Omega \mathrm{cm})^{-1}$.

The derivative $d R / d T$ shows a similar trend as previously observed in other samples, ${ }^{25-28}$ both in shape and in absolute magnitude; see the topmost inset in Fig. 4. The phase transition appears to be more distinct in the [110] directions, indicated by a slightly larger derivative.

The spin-polarized resistance $\Delta R_{\mathrm{sp}}$ was extracted by subtracting the measured resistance from a linear extrapolation (dotted lines in Fig. 4) as outlined in Ref. 28. Kats et al. suggested that $\Delta R_{\mathrm{sp}} \propto|t|^{2 \beta}$ where the reduced temperature $t=\left(T-T_{C}\right) / T_{C}$ and $\beta$ is the critical exponent of the magnetization. The logarithm of the spin-polarized resistance is shown in the lower right inset in Fig. 4 as a function of the logarithm of the reduced temperature $t$, where we have taken $T_{C}=145 \mathrm{~K}$. A linear trend is observed, consistent with the suggested behavior. The slope of this line gives the critical exponent $\beta \approx 0.34$ with only a small difference (0.003) between the [1 10] and [001] data. The critical exponent deduced from this data agrees well with the findings in Ref. 28.

\section{B. Magnetoresistance}

The low-temperature resistance as a function of applied magnetic field, shown in Fig. 5, exhibits a strong anisotropy.

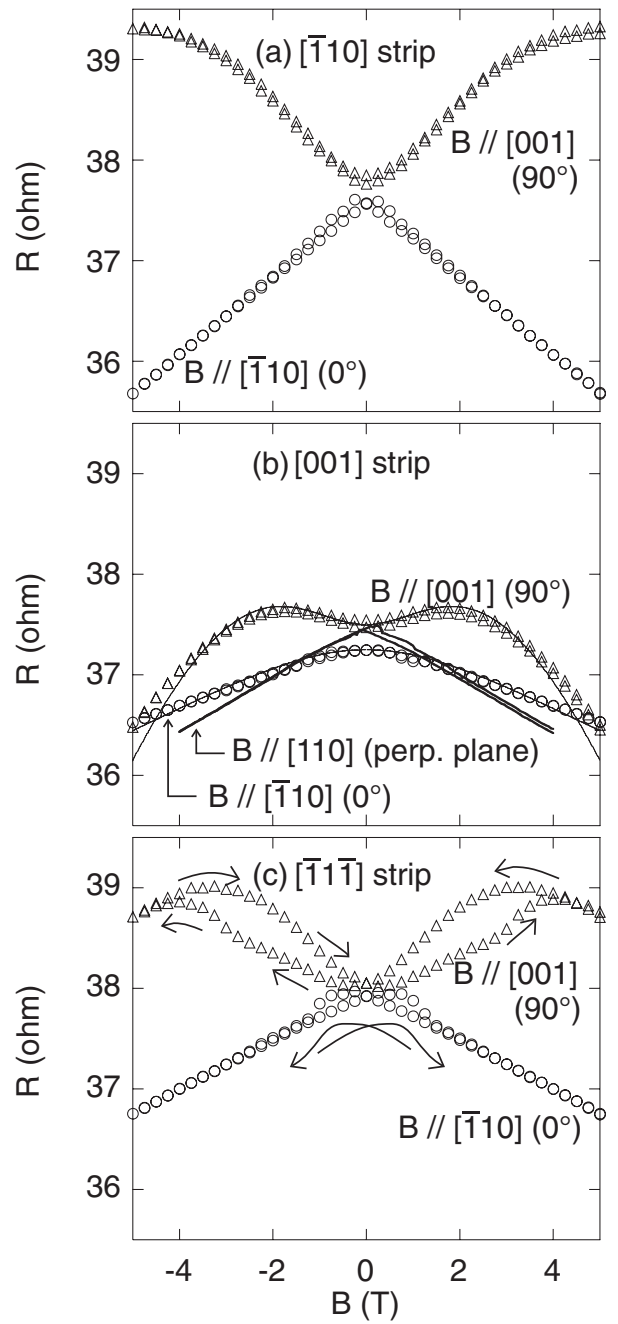

FIG. 5. Resistance at $2 \mathrm{~K}$ as a function of applied magnetic field with field applied in the [001] (open triangles) and [110] (open circles) directions. Data for the strips aligned along [1110], [001], and [1111] are shown in (a), (b), and (c), respectively. Solid line up to $\pm 4 \mathrm{~T}$ in (b) represents data with field applied perpendicular to the plane. Solid lines through triangles and circles are curves fitted with the antilocalization model from Ref. 4. The curves in (c) exhibit hysteresis as indicated by the arrows. The angles given in parentheses represent the direction of the applied magnetic field $\theta$ as defined in Fig. 3.

Not only is it different in the strips in different directions, but most prominent is the anisotropy with respect to the direction of the applied field. With the field parallel to [1 10] $\left(\theta=0^{\circ}\right)$ the magnetoresistance behaves quite differently from when the field is applied along the [001] $\left(\theta=90^{\circ}\right)$ direction. Whereas the former is negative and shows a close to linear behavior in $R(B)$ in the entire field range, the latter is generally positive and has a more rounded shape at low fields and becomes negative at high fields. One can note, however, that in the [1110] direction we observe the behavior only up to a maximum at $\pm 5 \mathrm{~T}$.

The general behavior of the magnetoresistance is nonhysteretic. However, in the $[\overline{1} 1 \overline{1}]$ strip a small hysteresis can be observed with the field in the [110] direction and a larger hysteresis with the field in the [001] direction. This is the largest hysteresis observed, and actually is only one measurement-the first field sweep after cool down. In the 


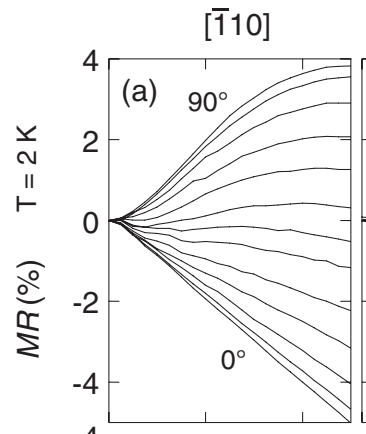

$[\overline{1} 1 \overline{1}]$
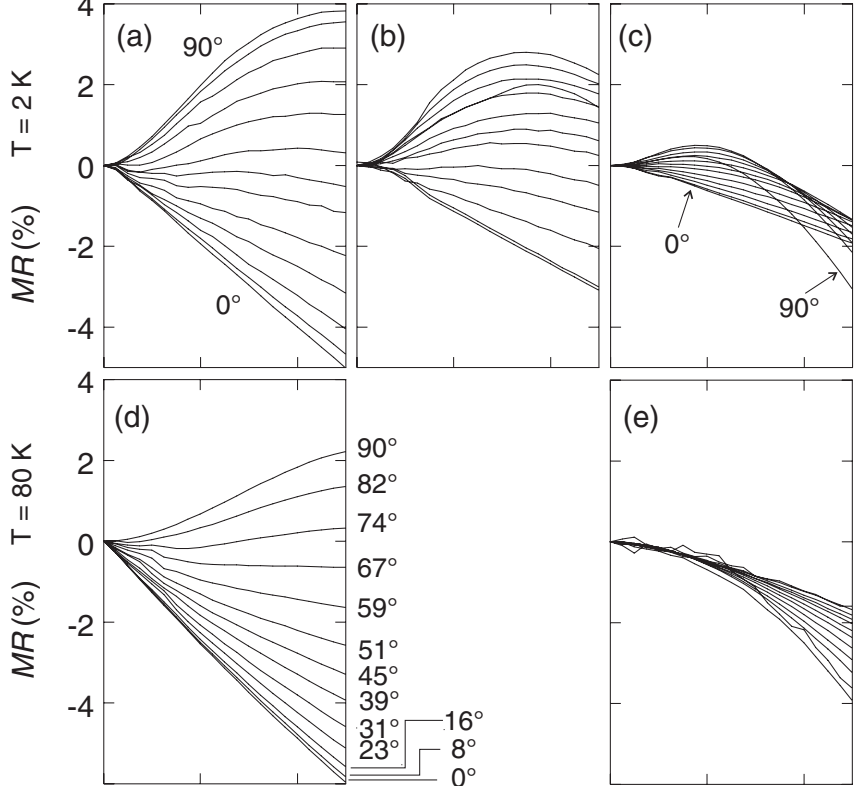

$90^{\circ}$
$82^{\circ}$

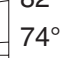

$67^{\circ}$

$59^{\circ}$

$51^{\circ}$
$45^{\circ}$

$45^{\circ}$
$39^{\circ}$

$31^{\circ}-16^{\circ}$

$\begin{array}{r}33^{\circ}-8^{\circ} \\ \hline\end{array}$
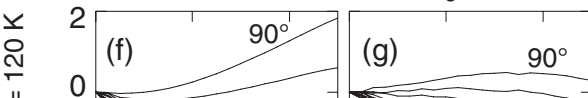

$90^{\circ}$

(h)

(e)
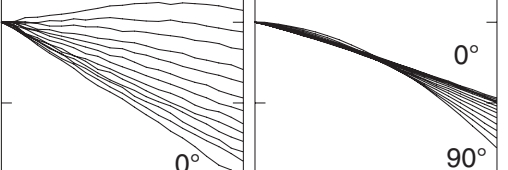

0.5

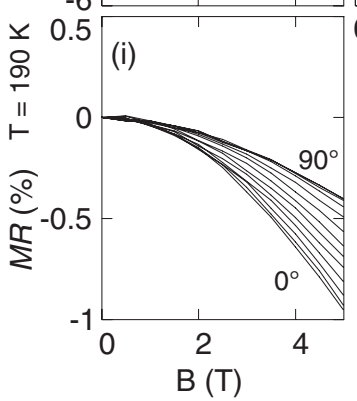

$\mathrm{B}(\mathrm{T})$
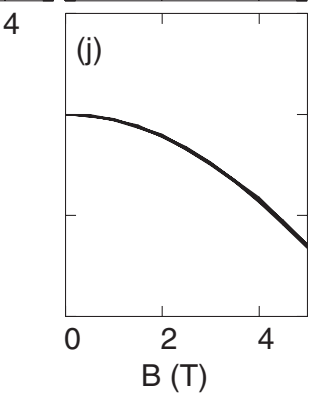

FIG. 6. Magnetoresistance $M R=[R(H)-R(0)] / R(0)$, measured with the field in different directions with respect to [110] $\left(0^{\circ}\right)$ : (a)-(c) at $2 \mathrm{~K}$, (d) and (e) at $80 \mathrm{~K}$, (f)-(h) at $120 \mathrm{~K}$, and (i) and (j) at 190 $\mathrm{K}$. The panels show data when the field was applied in the directions indicated between (d) and (e). Note that due to the curvature of the data lines the order is reversed in the high-field end of (h). The three columns of plots refer to data acquired from strips in the [110] (left column), [111]] (center column), and [001] (right column) directions.

other data (or after the initial field sweep) the hysteretic behavior was limited to within $\pm 1 \mathrm{~T}$.

The observation of both positive and negative magnetoresistance has been previously reported. ${ }^{6,17-19}$ However, in most cases it has been related to the relative direction of the current compared to the applied magnetic field. This is in contrast to the data presented here, where the positive magnetoresistance was observed at low fields in the [001] direction and negative magnetoresistance when the field was applied in the [110] direction. We therefore proceeded to map the magnetoresistance $M R=[R(H)-R(0)] / R(0)$ dependence on the field direction as shown in Fig. 6.

As the field is rotated from $0^{\circ}$ to $90^{\circ}$ there is a gradual transition from the linear negative magnetoresistance to a nonlinear positive magnetoresistance. The magnetoresistance with the field parallel to the [1110] direction appears to behave qualitatively similarly independent of current direction. Initially, at small fields the magnetoresistance increases (it is positive), reaching a maximum, decreases at a specific field, and eventually becomes negative. It appears as if the magnetoresistance in the $[\overline{1} 1 \overline{1}]$ direction is an intermediate state between [i110] and [001].

Data were collected at different temperatures $(2,80,120$, and $190 \mathrm{~K}$ ) where the latter is well above the Curie temperature. The magnetoresistance when the field is applied along [110] $\left(0^{\circ}\right)$ is larger in the [110] strip $(\sim-5 \%)$ than in the [001] strip $(\sim-2 \%)$, but is almost temperature independent, both in magnitude and in shape below $T_{C}$. At $190 \mathrm{~K}$, i.e., above $T_{C}$, the magnetoresistance is nonlinear and negative. In particular, there is a field direction dependence in the [110] strip whereas in the [001] strip the magnetoresistance curves collapse into a single trace [Fig. 6(j)].

One can define a characteristic field $H_{0}$ based on the field for the magnetoresistance maximum. Then at low temperature $(2 \mathrm{~K})$ we find $H_{0}^{[\overline{1} 10]} \approx 2 \mathrm{~T}, H_{0}^{[001]} \approx 5 \mathrm{~T}$, and $H_{0}^{[\overline{1} 11 \overline{1}]} \approx 3.5 \mathrm{~T}$. It appears that in the [110] strip the characteristic field increases with increasing temperature. However, as our experiments are limited to a maximum of $5 \mathrm{~T}$, we cannot determine $H_{0}$ for the higher temperatures. Also, for [ $1 \overline{1} 1 \overline{1}]$ the characteristic field increases slightly with temperature, from about $3.5 \mathrm{~T}$ at $2 \mathrm{~K}$ to closer to $4 \mathrm{~T}$ at $120 \mathrm{~K}$.

\section{DISCUSSION}

From structural and transport data (Figs. 2 and 4, respectively) we conclude that the performance of our samples is consistent with that of other high-quality samples. The general shape of the resistance versus temperature curves agrees well with results on samples from other groups. ${ }^{25,26}$ The resistivity kink at $T_{C}$ is consistent with a critical exponent of $\beta \approx 0.34$ independent of the direction of measurement ([ $[\overline{1} 10]$ or [001]). This would indicate that a three-dimensional Ising model could be used to describe the spontaneous magnetization. ${ }^{28,29}$

Our films also behave qualitatively in the same way as previously reported samples regarding the magnetotransport properties. The presence of both positive and negative magnetoresistance has previously been reported by Klein et al. (Fig. 6 in Ref. 6) and in $5 \mathrm{~nm}$ thin films by Ziese et al. (Fig. 12 in Ref. 18). A similar behavior has also been recorded in partially relaxed thin films measured in van der Pauw configuration. ${ }^{19}$ In general the data shown here resemble those data in many ways, e.g., the observation of a hysteresis particularly in the positive magnetoresistance.

The magnetoresistance has previously been shown to be a complicated function of temperature, $\operatorname{MR}(T),{ }^{6,27}$ actually quite similar to the temperature dependence of the planar Hall effect in $\mathrm{SrRuO}_{3} .{ }^{30}$ Here are presented more detailed combined measurements on the temperature and angular dependencies. These data suggest that at sufficiently high fields the magnetoresistance is always negative, but in fields parallel 
to the [001] direction the magnetoresistance is positive up to a critical field. The critical field appears to be stronger when the magnetoresistance is measured along [110] (i.e., perpendicular to [001]).

Typically, in quantum interference experiments one has to take the orientation of the magnetization and domain wall scattering into consideration, ${ }^{31}$ and indeed the magnetoresistance magnitude reported here is of the same order as previously reported domain wall resistivity in $\mathrm{SrRuO}_{3}{ }^{32}$ However, it appears as if the shape of the magnetoresistance is due to rotation of the magnetization direction rather than domain formation. The magnetoresistance does not die off as rapidly with increasing field as in Ref. 32. In magnetoresistance measurements the characteristic field for magnetic domains is the coercive field, and if we take the extraordinary Hall effect (not shown here) to reflect the magnetization of the sample we draw the conclusion from its hysteresis that the coercive field is less than $330 \mathrm{mT}$ (at $2 \mathrm{~K}$ ). We keep in mind that this method measures domain switching in the out-of-plane direction which is not along the low-energy direction of the magnetocrystalline anisotropy nor along an easily magnetized direction of the shape anisotropy. Therefore, the coercive field deduced from these measurements would most probably be an overestimate of the coercive field for the in-plane magnetization. This field coincides, however, quite well with the hysteretic peaks in the magnetoresistance of the [ $\overline{1} 1 \overline{1}]$ strip [the open circles in Fig. 5(c)]. In any case, this field is almost an order of magnitude smaller than $\mathrm{H}_{0}$.

We note that the easy axis in $\mathrm{SrRuO}_{3}$ is close to the $a$ or $b$ axis of the $\mathrm{SrRuO}_{3}$ unit cell depicted in Fig. 1(b) with a magnetocrystalline anisotropy field of $\sim 2 \mathrm{~T}$ (see Ref. 6 and Refs. 14, 23, and 24 therein). This anisotropy field is close to the characteristic field $H_{0}$ observed here. Therefore, at present we cannot completely rule out the conventional anisotropic magnetoresistance as explanation for the behavior of the measured data. On the other hand, anisotropic magnetoresistance is typically discussed in terms of the direction of the magnetic field relative to the direction of the current (see, e.g., Ref. 33) for epitaxial $\mathrm{SrRuO}_{3}$ films also (see, e.g., Refs. 17,34), which is inconsistent with our data. However, regardless of whether the shape of the magnetoresistance is due to weak antilocalization or to ordinary anisotropic magnetoresistance the spin-orbit interaction is at the root of both effects. But the temperature dependence of the anisotropic magnetoresistance is different in the two perpendicular directions, i.e., Figs. 6(a), 6(d), 6(f), and 6(i) show a different behavior than Figs. 6(c), 6(e), 6(h), and $6(\mathrm{j})$, and when $H_{0}$ appears to increase with increasing temperature in the [110] direction it appears to decrease with increasing temperature in the [001] direction. Hence, we can conclude that the spin-orbit interaction is anisotropic.

Spin-orbit coupling appears to be present when the field is applied in the [001] direction, whereas it seems that the spin-orbit coupling is absent (or too weak) when the field is applied in the [110] direction (only negative magnetoresistance). In addition, the data in Figs. 5 and 6 suggest that the effect of the anisotropic spin-orbit interaction is larger in the [110] direction-the maximum is positioned at higher field in this direction (perpendicular to the coupling itself). On the other hand we note that the overall magnitude of the magnetoresistance is larger in the same direction. It is reasonable to believe that these two effects, the magnitude of the magnetoresistance and the position of the maximum, are closely connected.

Using the slope that was measured for the ordinary Hall effect, and which is about $0.86 \pm 0.05 \mu \mathrm{V} / \mathrm{T}$ (at $100 \mu \mathrm{A}$ ), the charge carrier density is estimated as $n=1.5 \times 10^{22} \mathrm{~cm}^{-3}$. Together with $\rho$ this implies a mobility $\mu=1 /(\rho n e)=15$ $\mathrm{cm}^{2} /\left(\begin{array}{ll}\mathrm{V} & \mathrm{s}\end{array}\right)$. The electron diffusion coefficient $D$ is then given by the Einstein relation $D=\left(\frac{k_{B} T}{e}\right) \mu$ which at low temperature results in $D=2.5 \times 10^{-3} \mathrm{~cm}^{2} / \mathrm{s}$. Assuming this is a reasonable number, the characteristic field can be converted to scattering time via $\tau=\frac{\hbar}{e} \frac{1}{D H_{0}}$. Thus in the $[\overline{1} 10]_{\mathrm{SRO}}$ and $[001]_{\text {SRO }}$ directions we have $\tau_{\text {so }}^{[\overline{1} 10]} \approx 7.0 \times 10^{-13} \mathrm{~s}$ and $\tau_{\text {so }}^{[001]} \approx 2.8 \times 10^{-13} \mathrm{~s}$, respectively.

Our data fit qualitatively well to a model for weak antilocalization [Eq. (3.32) in Ref. 35], as shown in Fig. 5(b). The parameters in this model are the characteristic relaxation times for potential scattering $\left(\tau_{o}\right)$, inelastic scattering $\left(\tau_{i}\right)$, magnetic scattering $\left(\tau_{s}\right)$, and spin-orbit scattering $\left(\tau_{\text {so }}\right)$, respectively. The magnetoresistance data for the [001] and the [110] directions can be traced by varying only $\tau_{\text {so }}$. Hence, the fitting curves in Fig. 5(b) were generated by optimizing with respect to $\tau_{o}, \tau_{i}$, and $\tau_{s}$ and then keeping them fixed while finding $\tau_{\mathrm{so}}=3.5 \times 10^{-13} \mathrm{~s}$ and $\tau_{\mathrm{so}}=1.4 \times 10^{-13} \mathrm{~s}$ for the two perpendicular directions. (However, the exact values of $\tau_{\text {so }}$ depend on the other chosen scattering times.) We note that quantitatively these particular values for $\tau_{\mathrm{so}}$ are of the same order of magnitude and differ by only a small factor from the above estimated values, and qualitatively the model fits well to the measured data with the field in both the [001] and the [110] directions (both in plane). Traditionally, weak localization as well as weak antilocalization is considered in two-dimensional systems when a magnetic field is applied perpendicular to the plane. The data in this paper do not show any sign of weak antilocalization in the configuration perpendicular to the plane. However, there are several reports on the observation of localizationlike effects in various systems. ${ }^{36-38}$

In the in-plane configuration it is typically considered that the spin-dependent scattering takes place at surfaces. An in-plane magnetic field orders the orientation of the electron spins interacting with the surface, and the magneoresistance maximum occurs when the diffusion length associated with the spin-orbit scattering, $L_{\mathrm{so}}=\sqrt{D \tau_{\mathrm{so}}}$, is comparable to the magnetic length $L_{M}=\sqrt{\hbar /(e B)}$. The maximum then marks the separation between strong (low-field) and weak (highfield) spin-orbit interaction. ${ }^{36}$ The data shown here exhibit a variation of the strength of spin-orbit coupling with the crystalline direction. This could be associated with the band structure and the variation of the density of states in different directions, which is then in line with other systems (including oxides) where the strength of the spin-orbit coupling can be modified by tuning the charge carrier density (see, e.g., Refs. 39,40 and references therein). One might speculate if this could then be related to the very recently reported observation $^{41}$ of a scaling behavior of the anomalous Hall effect in $\mathrm{SrRuO}_{3}$ thin films.

Finally, we note that the anisotropic magnetoresistance observed in the paramagnetic state agrees well with the strongly angle-dependent induced magnetism in the paramagnetic state 
reported previously. ${ }^{34,42}$ A field-induced anisotropy has been reported in the paramagnetic temperature region. Now, if the spin-orbit interaction is anisotropic and its strength is field dependent, this could possibly also explain the anisotropic magnetoresistance above $T_{C}$.

In conclusion, this paper presents detailed information on the transfer from negative to positive magnetoresistance as the field is rotated in the sample plane. In addition, with the current along [001] there is a different temperature dependence of the magnetoresistance when the field is directed along [110] as compared to along [001]. Hence, the anisotropy data for these samples, and particularly for the single-orientation sample discussed here, indicate a different origin for the anisotropy than previously reported. The data presented here suggest that the magnetoresistance anisotropy could be connected to an anisotropic spin-orbit interaction and is a direct consequence of the orientation of the magnetic field with respect to the orthorhombic unit cell. The data show localizationlike effects; in particular, the data fit well to a weak antilocalization model.

\section{ACKNOWLEDGMENTS}

Tord Claeson and Alexey Kalabukhov are acknowledged for valuable comments and suggestions on the manuscript. Thanks also to Johan Börjesson, Chang-Beom Eom, Mikael Fogelström, Gervasi Herranz, and Eva Olsson for fruitful discussions on these data. The films were deposited by Khaled Khamchane, Alexey Kalabukhov, and Evgeny Stepantsov. *robert.gunnarsson@hlk.hj.se

${ }^{1}$ G. A. Prinz, Science 282, 1660 (1998).

${ }^{2}$ G. Bergmann, Phys. Rev. B 28, 2914 (1983).

${ }^{3}$ Yu. F. Komnik, V. V. Andrievski, and I. B. Berkutov, Low Temp. Phys. 33, 79 (2007).

${ }^{4}$ G. Bergmann, Int. J. Mod. Phys. B 24, 2015 (2010).

${ }^{5}$ V. J. Emery and S. A. Kivelson, Phys. Rev. Lett. 74, 3253 (1995).

${ }^{6}$ L. Klein, J. S. Dodge, C. H. Ahn, J. W. Reiner, L. Mieville, T. H. Geballe, M. R. Beasley, and A. Kapitulnik, J. Phys.: Condens. Matter 8, 10111 (1996).

${ }^{7}$ C. B. Eom, R. J. Cava, R. M. Fleming. J. M. Philips, R. B. van Dover, J. H. Marshall, J. W. P. Hsu, J. J. Krajewski, and W. F. Peck Jr., Science 258, 1766 (1992).

${ }^{8}$ P. Rundqvist, A. Vorobiev, S. Gevorgian, K. Khamchane, and Z. Ivanov, J. Appl. Phys. 93, 1291 (2003).

${ }^{9}$ A. P. Mackenzie, J. W. Reiner, A. W. Tyler, L. M. Galvin, S. R. Julian, M. R. Beasley, T. H. Geballe, and A. Kapitulnik, Phys. Rev. B 58, R13318 (1998).

${ }^{10}$ C. S. Alexander, S. McCall, P. Schlottmann, J. E. Crow, and G. Cao, Phys. Rev. B 72, 024415 (2005).

${ }^{11}$ L. Klein, J. R. Reiner, T. H. Geballe, M. R. Beasley, and A. Kapitulnik, Phys. Rev. B 61, R7842 (2000).

${ }^{12}$ Z. Fang, N. Nagaosa, K. S. Takahashi, A. Asamitsu, R. Mathieu, T. Ogasawara, H. Yamada, M. Kawasaki, Y. Tokura, and K. Terakura, Science 302, 92 (2003).

${ }^{13}$ R. Mathieu, C. U. Jung, H. Yamada, A. Asamitsu, M. Kawasaki, and Y. Tokura, Phys. Rev. B 72, 064436 (2005).

${ }^{14}$ G. Herranz, F. Sánchez, B. Martínez, J. Fontcuberta, M. V. GarcíaCuenca, C. Ferrater, and M. Varela, J. Appl. Phys. 95, 7213 (2004).

${ }^{15}$ M. Feigenson, L. Klein, J. W. Reiner, and M. R. Beasley, Phys. Rev. B 67, 134436 (2003).

${ }^{16}$ M. C. Langner, C. L. S. Kantner, Y. H. Chu, L. M. Martin, P. Yu, J. Seidel, R. Ramesh, and J. Orenstein, Phys. Rev. Lett. 102, 177601 (2009).

${ }^{17}$ R. A. Rao, D. B. Kacedon, and C. B. Eom, J. Appl. Phys. 83, 6995 (1998).

${ }^{18}$ M. Ziese, I. Vrejoiu, and D. Hesse, Phys. Rev. B 81, 184418 (2010).

${ }^{19}$ Yu. A. Boikov, and V. A. Danilov, Fiz. Tverd. Tela 53, 1230 (2011) [Phys. Solid State 53, 1298 (2011)].

${ }^{20}$ R. Gunnarsson, A. Kalabukhov, and D. Winkler, Surf. Sci. 603, 151 (2009).

${ }^{21}$ R. Gunnarsson, J. Börjesson, K. Khamchane, J. Holmlund, T. Claeson, and E. Olsson (unpublished).
${ }^{22}$ J. C. Jiang, W. Tian, X. Pan, Q. Gan, and C. B. Eom, Mater. Sci. Eng. B 56, 152 (1998).

${ }^{23}$ Q. Gan, R. A. Rao, C. B. Eom, L. Wu, and F. Tsui, J. Appl. Phys. 85, 5297 (1999).

${ }^{24}$ A. Vailionis, W. Siemons, and G. Koster, Appl. Phys. Lett. 93, 051909 (2008).

${ }^{25}$ L. Klein, J. S. Dodge, C. H. Ahn, G. J. Snyder, T. H. Geballe, M. R. Beasley, and A. Kapitulnik, Phys. Rev. Lett. 77, 2774 (1996).

${ }^{26}$ P. B. Allen, H. Berger, O. Chauvet, L. Forro, T. Jarlborg, A. Junod, B. Revaz, and G. Santi, Phys. Rev. B 53, 4393 (1996).

${ }^{27}$ L. M. Wang, H. E. Horng, and H. C. Yang, Phys. Rev. B 70, 014433 (2004).

${ }^{28}$ Y. Kats, L. Klein, J. W. Reiner, T. H. Geballe, M. R. Beasley, and A. Kapitulnik, Phys. Rev. B 63, 054435 (2001).

${ }^{29}$ M. Schultz, S. Levy, J. W. Reiner, and L. Klein, Phys. Rev. B 79, 125444 (2009).

${ }^{30}$ I. Genish, L. Klein, J. W. Reiner, and M. R. Beasley, Phys. Rev. B 75, 125108 (2007)

${ }^{31}$ S. Sil, P. Entel, G. Dumpich, and M. Brands, Phys. Rev. B 72, 174401 (2005).

${ }^{32}$ L. Klein, Y. Kats, A. F. Marshall, J. W. Reiner, T. H. Geballe, M. R. Beasley, and A. Kapitulnik, Phys. Rev. Lett. 84, 6090 (2000).

${ }^{33}$ I. A. Campbell, and A. Fert, in Ferromagnetic Materials, edited by E. P. Wohlfarth (North-Holland Publishing, Amsterdam, 1982), Vol. 3, p. 752.

${ }^{34}$ I. Genish, Y. Kats, L. Klein, J. W. Reiner, and M. R. Beasley, J. Appl. Phys. 95, 6681 (2004).

${ }^{35}$ G. Bergmann, Phys. Rep. 107, 1 (1984).

${ }^{36}$ Yu.F. Komnik, V. V. Andrievsskii, and I. B. Berkutov, Low Temp. Phys. 33, 79 (2007).

${ }^{37}$ F. J. Wong, R. V. Chopdekar, and Y. Suzuki, Phys. Rev. B 82, 165413 (2010).

${ }^{38}$ S. Sangiao, N. Marcano, J. Fan, L. Morellón, M. R. Ibarra, and J. M. de Teresa, Europhys. Lett. 95, 37002 (2011).

${ }^{39}$ M. Kohda, T. Bergsten, and J. Nitta, J. Phys. Soc. Jpn. 77, 031008 (2008).

${ }^{40}$ P. Zubko, S. Gariglio, M. Gabaay, P. Ghosez, and J.-M. Triscone, Annu. Rev. Condens. Matter Phys. 2, 141 (2011).

${ }^{41}$ N. Haham, Y. Shperber, M. Schultz, N. Naftalis, E. Shimshoni, J. W. Reiner, and L. Klein, Phys. Rev. B 84, 174439 (2011).

${ }^{42}$ Y. Shperber, I. Genish, J. W. Reiner, and L. Klein, J. Appl. Phys. 105, $07 \mathrm{~B} 106$ (2009). 